\title{
Advanced Friction Modeling in Sheet Metal Forming
}

\author{
J.Hol ${ }^{1, a}$, M.V. Cid Alfaro², T. Meinders ${ }^{3}$, J. Huétink ${ }^{3}$ \\ ${ }^{1}$ Materials innovation institute (M2i), P.O. box 5008, 2600 GA Delft, The Netherlands \\ ${ }^{2}$ Tata Steel Research - Development \& Technology, P.O. box 10000, 1970 CA IJmuiden, The \\ Netherlands \\ ${ }^{3}$ University of Twente, P.O. box 217, 7500 AE Enschede, The Netherlands \\ aj.hol@m2i.nl
}

Keywords: friction modeling, friction mechanisms, asperity contact, flattening, real contact area, ploughing, adhesion

\begin{abstract}
The Coulomb friction model is frequently used for sheet metal forming simulations. This model incorporates a constant coefficient of friction and does not take the influence of important parameters such as contact pressure or deformation of the sheet material into account. This article presents a more advanced friction model for large-scale forming simulations based on the surface changes on the micro-scale. When two surfaces are in contact, the surface texture of a material changes due to the combination of normal loading and stretching. Consequently, shear stresses between contacting surfaces, caused by the adhesion and ploughing effect between contacting asperities, will change when the surface texture changes. A friction model has been developed which accounts for these microscopic dependencies and its influence on the friction behavior on the macro-scale. The friction model has been validated by means of finite element simulations on the micro-scale and has been implemented in a finite element code to run large scale sheet metal forming simulations. Results showed a realistic distribution of the coefficient of friction depending on the local process conditions.
\end{abstract}

\section{Introduction}

Finite Element simulations of sheet metal products are everyday practice in the automotive industry. An accurate forming analysis can however only be made if, among others, the material behavior and friction conditions are modeled accurately. For material models, significant improvements have been made in the last decades, but in the majority of simulations still a simple Coulomb friction model is used. Consequently, it is still cumbersome to predict the draw-in and springback of a blank during forming processes correctly.

To better understand contact and friction conditions during lubricated sheet metal forming processes, experimental and theoretical studies have been performed. On microscopic level, friction is due to the adhesion and ploughing effect between contacting asperities and the appearance of hydrodynamic friction stresses. The real area of contact, which depends on different flattening and roughening mechanisms, plays an important role in characterizing friction. However, micro models encompassing these mechanisms are generally regarded as too cumbersome to be used in large scale simulations.

An advanced friction model is proposed which couples the most important friction mechanisms. Based on statistical parameters a fast and efficient translation from micro- to macro modeling is included. A general overview of the friction model is presented and the translation from micro to macro modeling is outlined. The development of real area of contact is described by the flattening models proposed by Westeneng [1] and the effect of ploughing and adhesion on the coefficient of friction is described by the friction model of Challen \& Oxley [1,2]. The flattening models are validated by means of FE simulations on micro-scale and the feasibility of the advanced macroscopic 
friction model is shown by a full scale sheet metal forming simulation.

\section{Theoretical background}

Solution procedure. A friction model, to be used in finite element codes, has been developed to couple the various micro friction models. The friction model starts with defining the process variables and material characteristics. Process variables are the nominal contact pressure and strain in the material which are calculated by the FE code. The contact force carried by the asperities equals the total nominal contact force since hydrodynamic friction stresses will not be accounted for. Significant material characteristics are the hardness of the asperities and the surface properties of the tool and workpiece material. Once the input parameters are known, the real area of contact is calculated based on the models accounting for flattening due to normal loading and flattening due to stretching. The amount of indentation of the harder tool asperities into the softer workpiece asperities can be calculated if the real area of contact and the contact pressure carried by the asperities are known. After that, shear stresses due to ploughing and adhesion effects between asperities and the coefficient of friction are calculated. It is noted that in reality flattening due to normal loading and flattening due to stretching will appear simultaneously during sheet metal forming, as well as the combination between flattening and sliding. Nevertheless, it has been assumed that the various mechanisms act independently of each other in this research.

Friction models encompassing micro-mechanisms are generally regarded as too cumbersome to be used in large-scale FE simulations. Therefore, translation techniques are necessary to translate microscopic contact behavior to macroscopic contact behavior. Using stochastic methods, rough surfaces are described on the micro-scale by their statistical parameters (mean radius of asperities, asperity density and the surface height distribution). Assuming that the surface height distribution on the micro-scale represents the surface texture on the macro-scale, it is possible to describe contact problems that occur during large-scale FE analyses of sheet metal forming processes [1].

Characterization rough surfaces. A discrete surface height distribution of the tool and workpiece material is obtained from surface profiles (Figure 1a). However, a continuous function is desirable to eliminate the need for integrating discrete functions during the solution procedure of the friction model. An advanced method to describe discrete signals can be achieved by using a Fourier series. A Fourier series makes it possible to describe non-smooth asymmetric distribution functions from which the accuracy of the evaluation depends on the number of expansions used.

The results discussed in this article are obtained by evaluating the surface height distribution func-

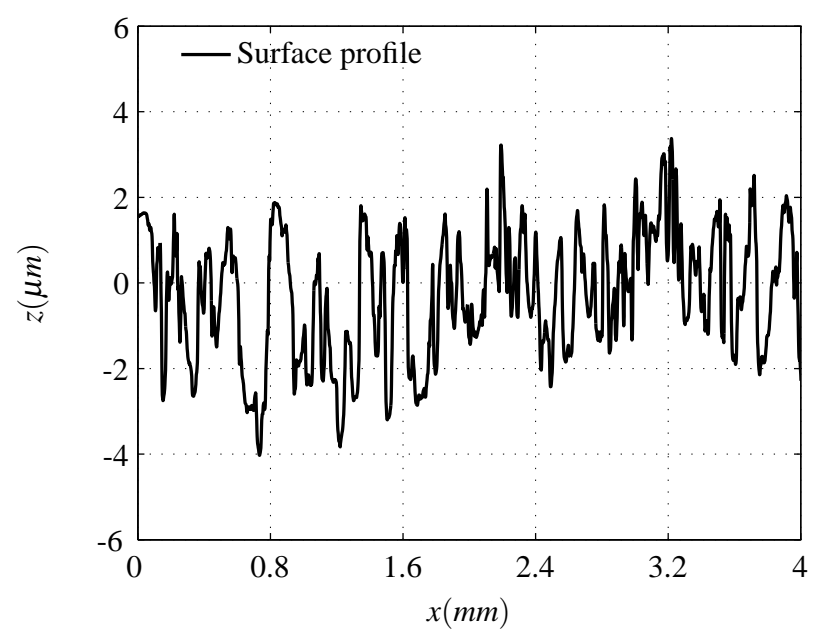

(a)

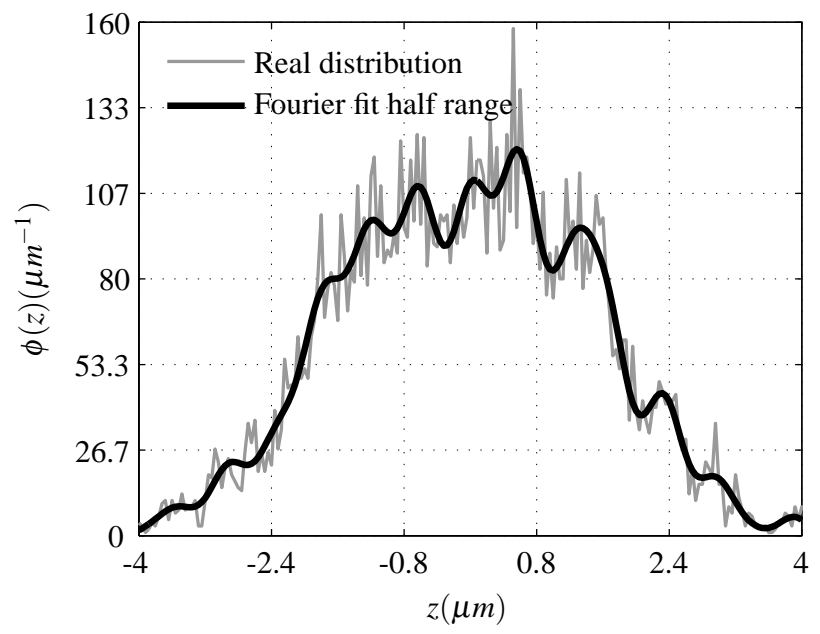

(b)

Figure 1: Surface profile (a) and corresponding surface height distribution (b) 
tions $\phi(z)$ by a half range sine Fourier function [3], given by:

$$
\phi(z)=\sum_{n=1}^{\infty} b_{n} \sin \left(\frac{n \pi}{L} z\right) \quad \text { with } \quad b_{n}=\frac{2}{L} \int_{0}^{L} f(z) \sin \left(\frac{n \pi}{L} z\right)
$$

in which $n$ represents the number of expansions, $L$ the evaluation domain and $f(z)$ the discrete form of the surface height distribution. In Figure 1b, the discrete surface height distribution from the workpiece material (Figure 1a) is evaluated by a Fourier function using 15 expansions.

Asperity flattening due to normal loading and stretching. Two flattening mechanisms have been implemented in the friction model to calculate the real area of contact of the workpiece: flattening due to normal loading and flattening due to stretching. In this section a brief explanation of the theory will be provided. For a detailed derivation of the following equations, the reader is referred to [4]. The asperities of the rough surface are modeled by bars which can represent arbitrarily shaped asperities. 3 stochastic variables are introduced: The normalized surface height distribution function of the asperities of the rough surface $\phi(z)$, the uniform rise of the non-contacting surface $U$ (based on volume conservation) and the separation between the tool surface and the mean plane of the asperities of the rough surface $d$. The following expression has been derived for the nominal contact pressure using energy and volume conservation laws:

$$
\begin{aligned}
& \frac{P_{\text {nот }}}{H}=\xi\left(1+\eta \chi \int_{d}^{\infty} \phi(z) \mathrm{d} z\right) \\
& U(1-\alpha)=\int_{d-U}^{\infty}(z-d) \phi(z) \mathrm{d} z \\
& \alpha=\int_{d-U}^{\infty} \phi(z) \mathrm{d} z
\end{aligned}
$$

The parameters $d$ and $U$ are calculated by simultaneously solving Equations 2, 3 and 4 . $P_{\text {nom }}$ represents the nominal contact pressure (input parameter) and $H$ the hardness. $\xi$ can be regarded as an energy factor and $\chi$ as a shape factor. The persistence parameter $\eta$ describes the amount of energy required to lift up the non-contacting asperities. A value of $\eta=0$ means that no energy is needed to rise the asperities, a value of $\eta=1$ implies that a maximum amount of energy is needed to rise the asperities.

The above equations are based on a normal loading case without additional bulk strain. To account for flattening due to stretching, the models have to be adapted. The change of the fraction of the real contact area as a function of the nominal strain can be presented as:

$$
\frac{\mathrm{d} \alpha_{S}^{n}}{\mathrm{~d} \varepsilon}=\frac{l}{E} \phi\left(d_{S}^{n-1}-U_{S}^{n-1}\right)
$$

with $n$ the iteration number. The subscript $S$ is used for variables that become strain dependent. The contact area ratio is updated incrementally by:

$$
\alpha_{S}^{n}=\alpha_{S}^{n-1}+\mathrm{d} \alpha_{S}^{n}
$$

The initial values $\alpha_{S}^{0}, d_{S}^{0}$ and $U_{S}^{0}$ are obtained from the model without bulk strain. To calculate the change of $\alpha_{S}$, the value of $U_{S}$ and $d_{S}$ needs to be solved simultaneously while $\varepsilon$ is incrementally increased. Based on volume conservation (Equation 8) and the definition of the fraction of real contact area (Equation 7) $U_{S}$ and $d_{S}$ can be obtained.

$$
\begin{aligned}
& \alpha_{S}=\int_{d_{S}-U_{S}}^{\infty} \phi(z) d z \\
& U_{S}\left(1-\alpha_{S}\right)=\int_{d_{S}-U_{S}}^{\infty}\left(z-d_{S}\right) \phi(z) d z
\end{aligned}
$$


Shear stresses. The model of Challen \& Oxley $[2,5]$ takes the combining effect of ploughing and adhesion between a wedge-shaped asperity and a flat surface into account. Westeneng [1] extended the model of Challen \& Oxley to describe friction conditions between a flat workpiece material and multiple tool asperities. He assumed that the flattened peaks of the asperities are soft and perfectly flat and the surface of the tool material is rough and rigid. The difference in hardness between the tool and workpiece material and the difference in length scales between the two surfaces is significant in the case of a sheet metal forming process. Therefore, it is valid to make a subdivision in two length scales using a rigid tool and a soft workpiece. The 'macro-scale' model of Challen \& Oxley has been implemented in the friction model to describe friction conditions between the tool and workpiece material:

$$
F_{w}=\rho_{t} \alpha_{S} A_{\text {nom }} \int_{\delta}^{s_{\max }} \mu_{\text {asp }} \pi \omega \beta_{t} H \phi_{t}(s) d s
$$

with $\omega$ the amount of indentation, $\beta_{t}$ the mean radius of the tool asperities and $\mu_{\text {asp }}$ the coefficient of friction at single asperity scale [5]. $\rho_{t}$ represents the asperity density of the tool surface, $A_{n o m}$ the nominal contact area and $\phi_{t}$ the normalized surface height distribution function of the tool surface. The bounds of the integral are described by $s_{\max }$, the maximum height of the tool asperities, and $\delta$, the separation between the workpiece surface and the mean plane of the tool asperities. Since the normal force is known (input parameter), the coefficient of friction can finally be obtained by:

$$
\mu=\frac{F_{w}}{F_{N}}
$$

\section{Validation flattening models}

The flattening models proposed by Westeneng are used to determine the real area of contact between the tool and workpiece material. FE simulations on the micro-scale have been performed in order to validate these models. Two sets of simulations have been performed for this purpose. In the first analysis, a two-dimensional rough surface of $4 \mathrm{~mm}$ long was indented by a perfectly flat and rigid tool. The second analysis was focused on indenting a rough surface by a normal load including a bulk strain in the underlying material. Three simulations have been executed for each analysis using different roughness profiles. The roughness profiles used corresponds to three roughness measurements on DC04 low-carbon steel. The surface height distributions used for the analytical model belongs to the roughness profiles used for the FE simulations. A fixed hardness of $450 \mathrm{MPa}\left(3 \sigma_{y}\right)$ was used in the analytical model since a yield strength of $150 \mathrm{MPa}$ was used for the FE simulation.

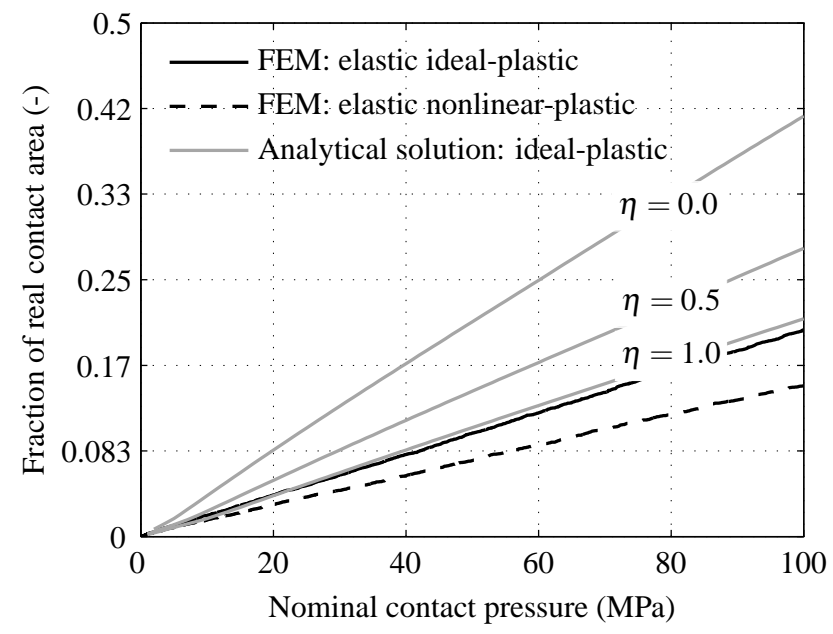

(a)

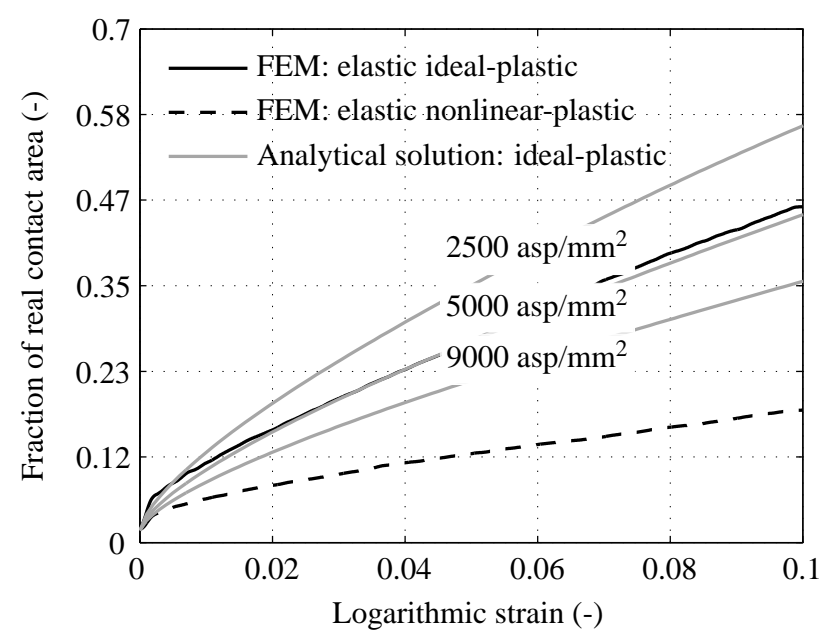

(b)

Figure 2: Development real contact area of analysis 1 (a) and development contact area of analysis 2 (b) 
The development of the real area of contact has been tracked during the simulations and compared with the analytical solutions. The analytical solutions and the FE solutions for elastic ideal-plastic and elastic non-linear plastic material behavior are presented in Figure 2. The results shown are the mean values of the three simulations performed per analysis case. As can be seen in Figure 2, taken work hardening effects into account has a significant influence on the development of the real area of contact. Due to work hardening, material subjected to high strains will harden resulting a higher resistance against asperity flattening. Consequently, taken work hardening into account results in a lower development of the real contact area.

The analytical model to describe flattening due to normal loading (Figure 2a) uses a persistence parameter $\eta$ to describe the amount of energy required to lift up the non-contacting asperities [1]. A value of $\eta=0$ means that no energy is required to rise the asperities, whereas a value of $\eta=1$ implies that a maximum amount of energy is required to rise the asperities. Since the exact value of this parameter is not known, different calculations have been performed in order to obtain a precise value for this parameter. A higher value for the persistence parameter $\eta$ results a smaller value of the real contact area (Figure 2a). The real contact area calculated by the analytical solution using a value of $\eta=1$ correspond very well to the elastic ideal-plastic FE solution. The analytical model deviates from the more realistic elastic nonlinear-plastic FE simulation, since work-hardening effects are not accounted for. The flattening of the asperities will be lower due to work-hardening effects, which in turn result in a lower amount of real area of contact (Figure 2a).

Combined normal loading and stretching the underlying bulk material decreases the effective hardness [6]. A lower hardness results in an increase of the real area of contact. Both the analytical and the FE results of analysis 2, where a rough surface has been indented by a nominal load and a bulk strain has been applied to the underlying material, are presented in Figure $2 \mathrm{~b}$. As for analysis 1 , results shown are the mean values of the three simulations performed per analysis case. It can be concluded from Figure $2 b$ that work-hardening effects have a large influence on the flattening behavior of the asperities. A difference of $20 \%$ in the real area of contact is obtained at the end of the simulation between the results of the elastic ideal-plastic and the elastic nonlinear-plastic simulation.

The density of workpiece asperities (in $\mathrm{mm}^{-2}$ ) is an unknown parameter in the analytical strain model. Hence, calculations have been performed using realistic values for the asperity density for DC0 4 to show the importance of this parameter, see Figure 2b. From this figure, it can be concluded that the asperity density of the workpiece has a significant influence on the development of the real area of contact. If a higher value of the asperity density is used the amount of real area of contact will be lower. The trend of the graphs corresponds well to the flattening behavior obtained by the FE simulations. Using an asperity density of $5000 \mathrm{asp} / \mathrm{mm}^{2}$ it is possible to describe the results of the elastic ideal-plastic FE solution (which has comparable material characteristics) precisely.

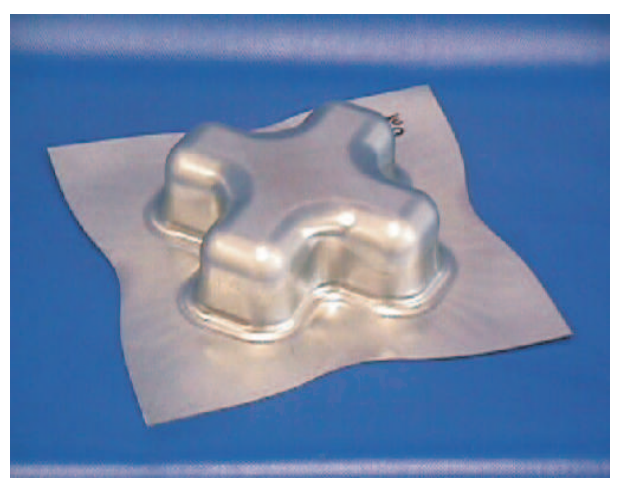

Table 1: Roughness parameters

\begin{tabular}{lll} 
Roughness parameter & Value & Unit \\
\hline \hline Hardness workpiece $(H)$ & 1400 & $\mathrm{MPa}$ \\
Persistence parameter $(\eta)$ & 1 & \\
Density workp. asp. $\left(\rho_{\text {work }}\right)$ & $5.0 \cdot 10^{3}$ & $\mathrm{~mm}^{-2}$ \\
Density tool. asp. $\left(\rho_{\text {tool }}\right)$ & $2.0 \cdot 10^{3}$ & $\mathrm{~mm}^{-2}$ \\
Radius tool. asp. $\left(\beta_{\text {tool }}\right)$ & $2.0 \cdot 10^{-2}$ & $\mathrm{~mm}$ \\
Nr. of Fourier expansions & 10 & \\
\hline
\end{tabular}

Figure 3: Example cross-die product 


\section{Application}

The cross-die product is a test piece designed at Renault which approximates process conditions of complex automotive parts (Figure 3). The cross-die product is used to test the numerical performance of the developed friction model in a large-scale FE simulation. To validate the model, an experimental test procedure is currently being setup.

Due to symmetry of the cross-die product only a quarter of the workpiece was modeled. The workpiece was meshed by 9000 triangular discrete Kirchhoff shell elements using 3 integration points in plane and 5 integration points in thickness direction. The yield surface was described by the Vegter model [7] using the Bergström-Van Liempt hardening relation [8] to describe hardening behavior. Material parameters were used from DC04 low carbon steel, a typical forming steel used for SMF processes. Contact between the tools and the workpiece was described by a penalty method using a penalty stiffness of $200 \mathrm{~N} / \mathrm{mm}$. The coefficient of friction used in the contact algorithm was calculated on the basis of the friction model presented in this article. Roughness parameters are given in Table 1. The simulation was performed by prescribing the displacement of the punch until a total displacement of $50 \mathrm{~mm}$ was reached. The punch speed was set to $5 \mathrm{~cm} / \mathrm{sec}$ and the force applied to the blankholder

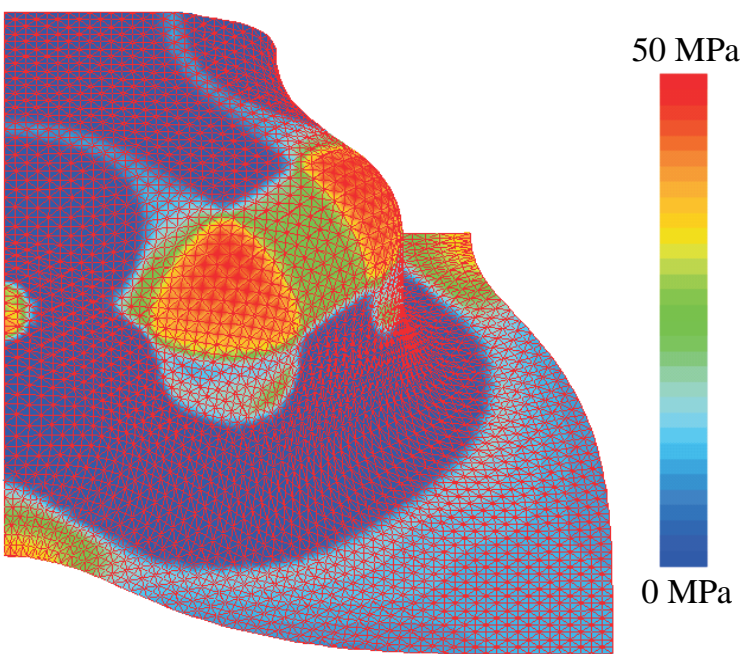

(a)
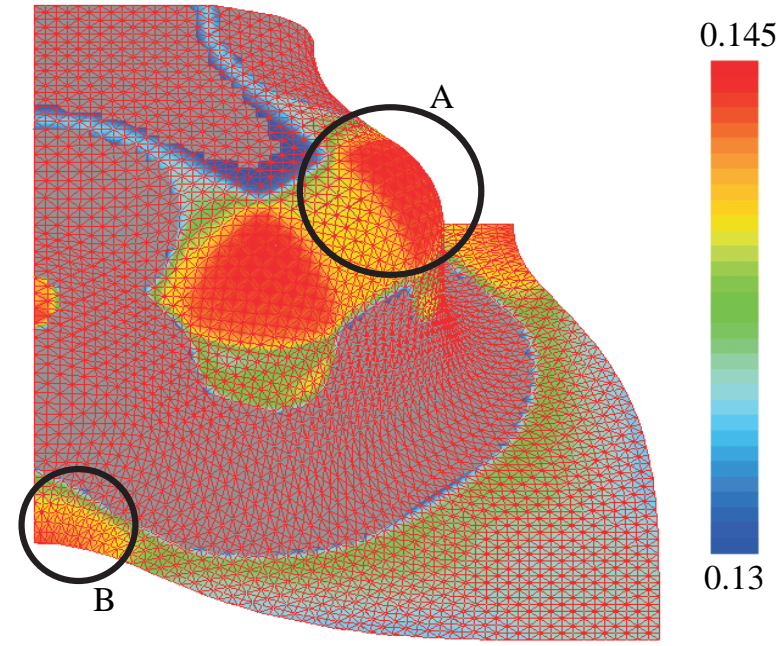

(b)

Figure 4: Development of contact pressure (a) and coefficient of friction (b) for normal loading only (gray represents the non-contacting area)

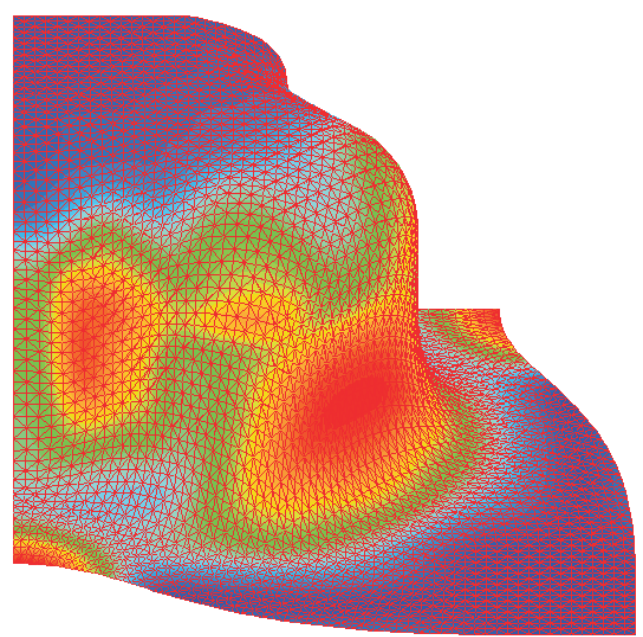

(a)

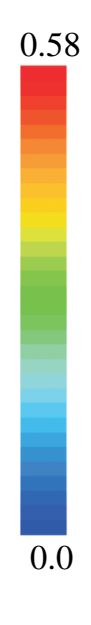

Figure 5: Development of equivalent plastic strain (a) and coefficient of friction (b) for normal loading + stretching (gray represents the non-contacting area) 
was $50 \mathrm{kN}$.

Two simulations have been performed in order to quantify the individual contributions of the two flattening mechanisms. The first simulation only accounted for the influence of normal loading on the coefficient of friction, Figure 4. The second simulation uses both flattening models to determine the coefficient of friction, Figure 5. Results shown in Figure 4 and 5 are from the punch side of the sheet. The gray areas represent the non-contacting areas.

If only flattening due to static loading is assumed (Figure 4), rather low values for the ratio of the real to the nominal area of contact are obtained. This results in friction coefficients that vary between 0.13 and 0.145 . Higher values are obtained in high-pressure regions: the contact area of the punch radius (region $\mathrm{A}$ ) and the thickened area of the blankholder region (region B). Lower values occur in low-pressure regions: the blankholder region and the top area of the punch. Results look reasonable, but it should be noted that only one of the two flattening mechanisms was taken into account during the simulation. If the second flattening mechanism is taken into account (flattening due to stretching), higher values for the real area of contact are obtained (Figure 5). The higher contact ratios result in higher values of the coefficient of friction, i.e. between 0.13 and 0.19. It can be observed from Figure 5 that higher values of the coefficient of friction occur at regions where high strains occur. Region $\mathrm{C}$ is purely stretched, region $\mathrm{D}$ is compressed which causes thickening of the material and region $\mathrm{E}$ is stretched over the die radius. On the other hand, low values of the coefficient of friction can be observed in low-strain regions. Overall it can be concluded that the distribution of the coefficients of friction lies within the range of expectation.

\section{Conclusions}

A friction model that can be used in large-scale FE simulations is presented. The friction model includes two flattening mechanisms to determine the real area of contact at a microscopic level. The real area of contact is used to determine the influence of ploughing and adhesion effects between contacting asperities on the coefficient of friction. A statistical approach is adapted to translate the microscopic models to a macroscopic level.

The friction model has been validated by means of FE simulations at a micro-scale. An excellent comparison between the analytical and the FE simulation is obtained in case of indenting a rough surface by a normal load. It was also found that work-hardening effects do not play a significant role in the case of pure normal loading. If a nominal strain is applied to the bulk material, the effect of workhardening becomes much more significant. The analytical model is able to accurately describe the FE results based on a elastic ideal-plastic material model. However, it was not possible to accurately describe the influence of work-hardening effects due to the large difference between the elastic idealplastic FE results and the elastic nonlinear-plastic FE results. It is concluded that work-hardening effects should not be neglected in the analytical strain model.

The friction model has been implemented in a FE code and applied to a full-scale sheet metal forming simulation. Results of the simulations have shown reasonable values for the coefficient of friction in the case of normal loading only, namely between 0.13 and 0.145 . If flattening due to stretching is also incorporated, more realistic values are achieved (between 0.13 and 0.19 ).

\section{Acknowledgment}

This research was carried out under the project number MC1.07289 in the framework of the Research Program of the Materials innovation institute M2i (www.m2i.nl).

\section{References}

[1] J. Westeneng, Modelling of contact and friction in deep drawing processes, Ph.D. thesis, University of Twente, 2001.

[2] J. Challen, P. Oxley, Wear 53 (1979) 229-243. 
[3] M. Greenberg, Advanced Engineering Mathematics - 2nd edition, Prentice Hall, 1998.

[4] J. Hol, M. C. Alfaro, M. de Rooij, T. Meinders, WEAR (submitted in 2010).

[5] J. Challen, P. Oxley, International Journal of Mechanical Siences 26 (1984) 403-418.

[6] W. Wilson, S. Sheu, International Journal of Mechanical Science 30 (1988) 475-489.

[7] H. Vegter, A. van den Boogaard, International Journal of Plasticity 22 (2006) 557-580.

[8] A. van den Boogaard, J. Huétink, Computer Methods in Applied Mechanics and Engineering 195 (2006) 6691-6709. 